\title{
Features Fusion based on the Fisherface and Simplification of the Laplacian Smoothing Transform
}

\author{
Arif Muntasa \\ Computational Artificial Intelligence Laboratory \\ Informatics Engineering Department, Engineering Faculty, University of Trunojoyo-Madura \\ Ry Telang Po. Box 2 Kamal, Bangkalan, Indonesia \\ arifmuntasa@trunojoyo.ac.id
}

\begin{abstract}
The newest model was proposed to extract the characteristic as distinctive attribute based on the Simplification of the Laplacian Smoothing Transform (S-LST) and the Fisherface. The proposed model is composed of two primary processes, i.e., training and testing processes. A training process involved features extraction based on the S-LST and the Fisherface, selection of the principal features, and features fusion of them. The proposed model has proved that the features can preserve the particular global information and the local feature areas of the rows and columns. The testing process is the stage to obtain the primary features through the new projection of the training sets. The last operation of the testing process is to measure the similarity of the features. The proposed model was tested using three different databases, i.e., the ORL, the YALE, and the UoB. The proposed model demonstrated the best performance, which is $100 \%$ recognition rate on the ORL and YALE when five images are implemented to the training sets. The maximum average of the recognition rate is $98.7 \%$ for the ORL database, $97.7 \%$ for the YALE database, and $94.53 \%$ for the UoB database. The comparison result showed that the proposed model performs better than the other approach, i.e., LST+LDA, S-MFA, S-NPE, S-LPP, S-LDA, LPP, LDA, and PCA.
\end{abstract}

Keywords: Extract the Feature, Features Fusion, Simplification of the Laplacian Smoothing Transform, and Fisherface.

\section{Introduction}

Computer vision has been trending topics in many sectors, such as security, government, company, and event academic fields. Some research results have been implemented in these areas. However, the results still also developed to overcome the limitations. The crucial problem is high dimensionality in similarity measurements. Many approaches have been promoted to overcome the problems, i.e. Covariance-based subspace [1], Eigenface [2-4], Fisherface [5-9], Independent Component Analysis [10], Local manifold model [11-12], Laplacian Smoothing Transform (LST) [13-14], Kernel subspace [15], Tangent Space [16], Sparse Neighborhood [17], Wavelet [18], Graph embedding [19], Matrix-based Features [20], Vertical and Horizontal Information [21], Locally Linear Regression [22], and Homogeneous and Non-homogeneous Polynomial Model [23].

PCA is global structure model to extract the image features [2-4]. The PCA is still referred and developed by many researchers, because of the simplicity. The PCA only needs small storage to represents the new spaces and their weights. Therefore, it does not require the larger computation. However, the PCA has fundamental limitations, firstly, a dependence of feature extraction results in dimensionality to some the training sets. Secondly, the PCA cannot capture the simplest invariant of the object, unless it explicitly represented the information. Lastly, the covariance matrix is hard to evaluate accurately.

Linear Discriminant Analysis (LDA) is the extension of the PCA. The LDA can preserve the sensitive information. Therefore, it can reduce the error rate when classification process is performed [5-9]. However, the LDA has also weaknesses, i.e., the correlation pattern was interpreted the similar from a group to each other, the function of discriminant built has a normal

Received: June $19^{\text {th }}, 2017$. Accepted: December $27^{\text {th }}, 2017$

DOI: 10.15676/ijeei.2017.9.4.6 
distribution for each group being compared. The LPP can preserve the local structure unless it has linear technique. Therefore, it spent less time than the non-linear model. The LST is a linear technique to reduce the features. However, it has a weakness, i.e., it cannot preserve the particular global information and the local feature areas for both the rows and columns. In this research, features fusion of Simplification of the Laplacian Smoothing Transform (S-LST) and Fisherface is proposed. The proposed model is quite different to the LST+LDA [13]. On the LST+LDA, the new projections were directly processed to the LST, whereas the proposed model has improved the LST, it is called S-LST. Furthermore, the Fisherface was independently processed to acquire the new spaces. The results of them were combined into the single unit feature. The proposed model has the advantage of representing the main features compared with LST, for the proposed model has been integrating information both horizontal and vertical an image. The features used of the proposed model were also smaller than the LST, but it produced the higher acceptance rates the other methods, included the LST.

The paper is composed of five sections. The first section, "Introduction", in this section, some popular methods were discussed, for both advantages and their disadvantages. The second section, the "Proposed Approach" is presented in detail. This part elucidated the S-LST, Fisherface, and features fusion. The third part, "Databases and Scenarios", where it presented the database image used to experiment based on the scenario. Furthermore, "Experimental Results and Discussions", this section demonstrates the results for each scenario and its analysis. "The conclusion" is written in the last section. This section resumes the analysis results of the proposed model.

\section{Proposed Approach}

Three main stages were proposed to improve the LST, i.e., firstly is feature extraction of the training sets. It consists of five primary processes as demonstrated in Figure 1, which are the SLST, the Fisherface, selection of the principal features of the S-LST, selection of the main features of the Fisherface, and features fusion. Secondly is feature extraction of the testing sets as displayed in Figure 2, it also consists of five primary processes as mentioned on the feature extraction of the training sets, but there are some differences of the detail processes. The last is the similarity measurement as shown the end of operation in Figure 2.

\section{A. Features Extraction based on the S-LST}

Feature extraction is the critical issue in computer vision. Massive dimensionality has been an exciting problem for an object detection, classification and also identification. The best solution to overcome the above problem is feature extraction. The feature extraction is a set of methods to capture the primary features of the object. It is performed to reduce the computation time when the similarity measurement process is completed. In this research, feature fusion of the S-LST and the Fisherface was proposed. Feature extraction is started with the S-LST process, where the S-LST has four major operations, i.e., compute the optimum value of the LST, S-LST, calculate the Eigenvalues and Eigenvectors, and determine the new spaces of the S-LST.

\section{B. Compute the optimum value of the LST}

Suppose height and width represent height and width of the image, whereas the dimensional of the LST is $n$, where $n=h e i g h t \times$ width. The LST matrix can be constructed using the identity matrix of size $n$ as the multiplication result of the columns and rows, and then the values of " 1 " are placed on the (row, row +1$)$ and $($ row $+1,1)$ as follows

$$
\begin{aligned}
& L_{1}(\text { row }, \text { row }+1)=1 \\
& L_{1}(\text { row }+1, \text { row })=1
\end{aligned}
$$

The element of "1" values in the diagonal matrix should be shifted to the right and bottom to obtain these values as mentioned in Equation (1) as the following pseudo-code 


$$
\begin{aligned}
& \text { row } \leftarrow 0 \\
& \text { while } \text { row }<n \text { do } \\
& \text { row } \leftarrow \text { row }+1 \\
& \text { col } \leftarrow 0 \\
& \text { while } c o l<n \text { do } \\
& \quad \text { col } \leftarrow \text { col }+1 \\
& \quad L_{1}(\text { row }, \text { col }) \leftarrow 0
\end{aligned}
$$

End while of $\mathrm{col}$

$$
\text { End while of row }
$$

row $\leftarrow 0$

while $r$ ow $<\mathrm{n}-1$ do

$$
\begin{aligned}
& \text { row } \leftarrow \text { row }+1 \\
& L_{1}(\text { row }, \text { row }+1) \leftarrow 1 \\
& L_{1}(\text { row }+1, \text { row }) \leftarrow 1
\end{aligned}
$$

End while of row

Pseudo-code 1. It is used to create $L_{1}$

The $L_{1}$ matrix elements should be changed to zero value on the particular location. It is utilized to obtain and divide the information areas of the object as the following equation

$$
\begin{aligned}
& L_{1}(\text { height } \times \text { row }+1, \text { height } \times \text { row })=0 \\
& L_{1}(\text { height } \times \text { row, height } \times \text { row }+1)=0
\end{aligned}
$$

The Equation (2) can be easily applied by using pseudo-code as follows

row $\leftarrow 0$

while row $<$ width

$$
\begin{aligned}
& \text { row } \leftarrow \text { row }+1 \\
& L_{1}(\text { height } \times \text { row }+1 \text {, height } \times \text { row }) \leftarrow 0 \\
& \left.L_{1} \text { (height } \times \text { row, height } \times \text { row }+1\right) \leftarrow 0
\end{aligned}
$$

End while of row

Pseudo-code 2. Replace certain element of the $L_{1}$

The matrix result as written in Pseudocode 1 and 2 were used to sharpen and preserve the global particular information areas of the object. It must be replaced with the " 1 " values on the precise location as shown in Equation (3)

$$
\begin{aligned}
& L_{1}(\text { row }, \text { row }+ \text { height })=1 \\
& L_{1}(\text { row }+ \text { height }, \text { row })=1
\end{aligned}
$$

The Equation (3) is applied through shifting of the " 1 " values to the right and the bottom as much as the height of an image as the following pseudo-code 
Arif Muntasa

row $\leftarrow 0$

while row $<n$-height do

row $\leftarrow$ row +1

$L_{1}($ row , row + height $) \leftarrow 1$

$L_{1}($ row + height, row $) \leftarrow 1$

End while of row

Pseudo-code 3. It is used to create $L_{1}$

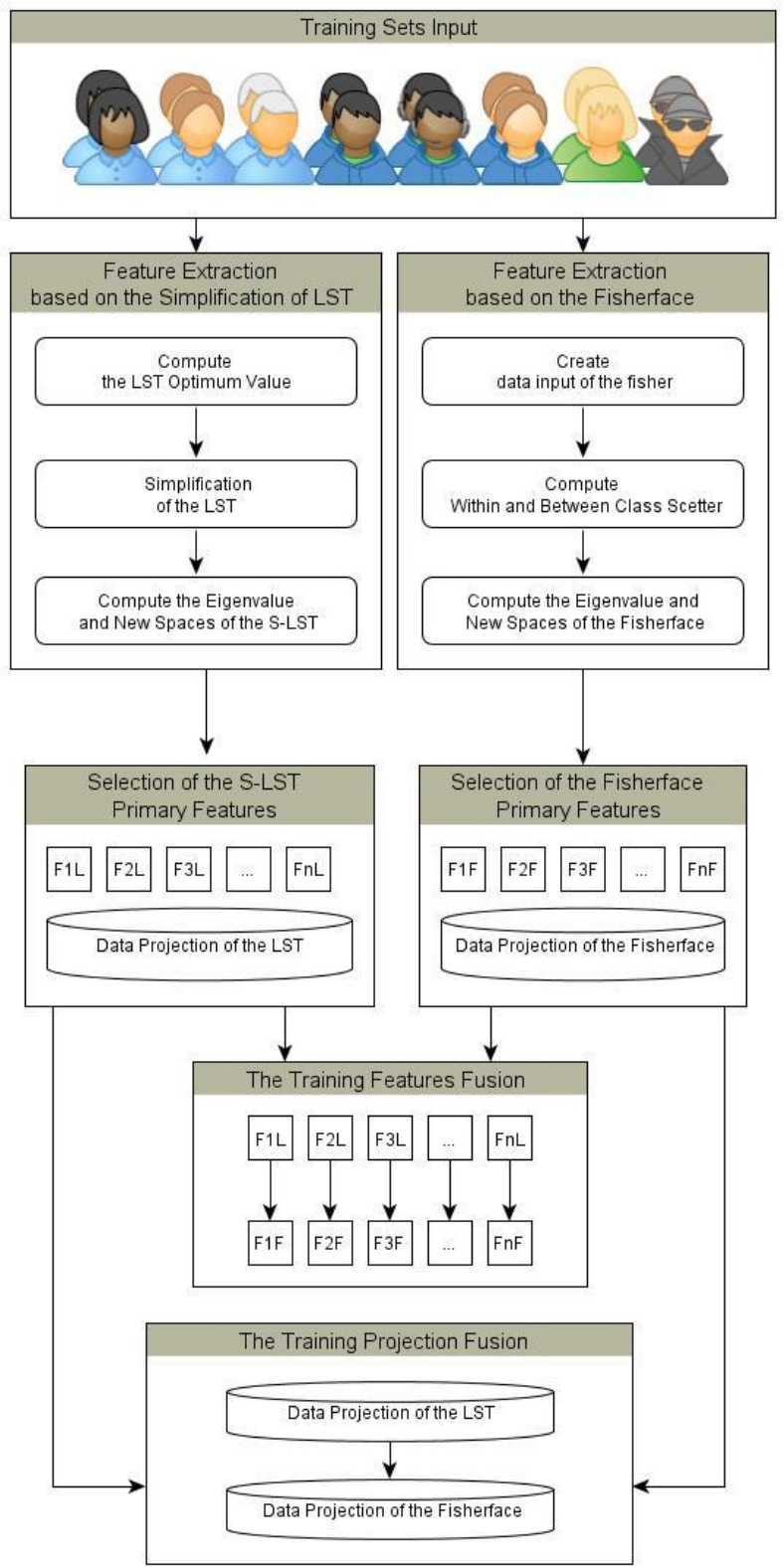

Figure 1. Training Process of the Proposed Model 


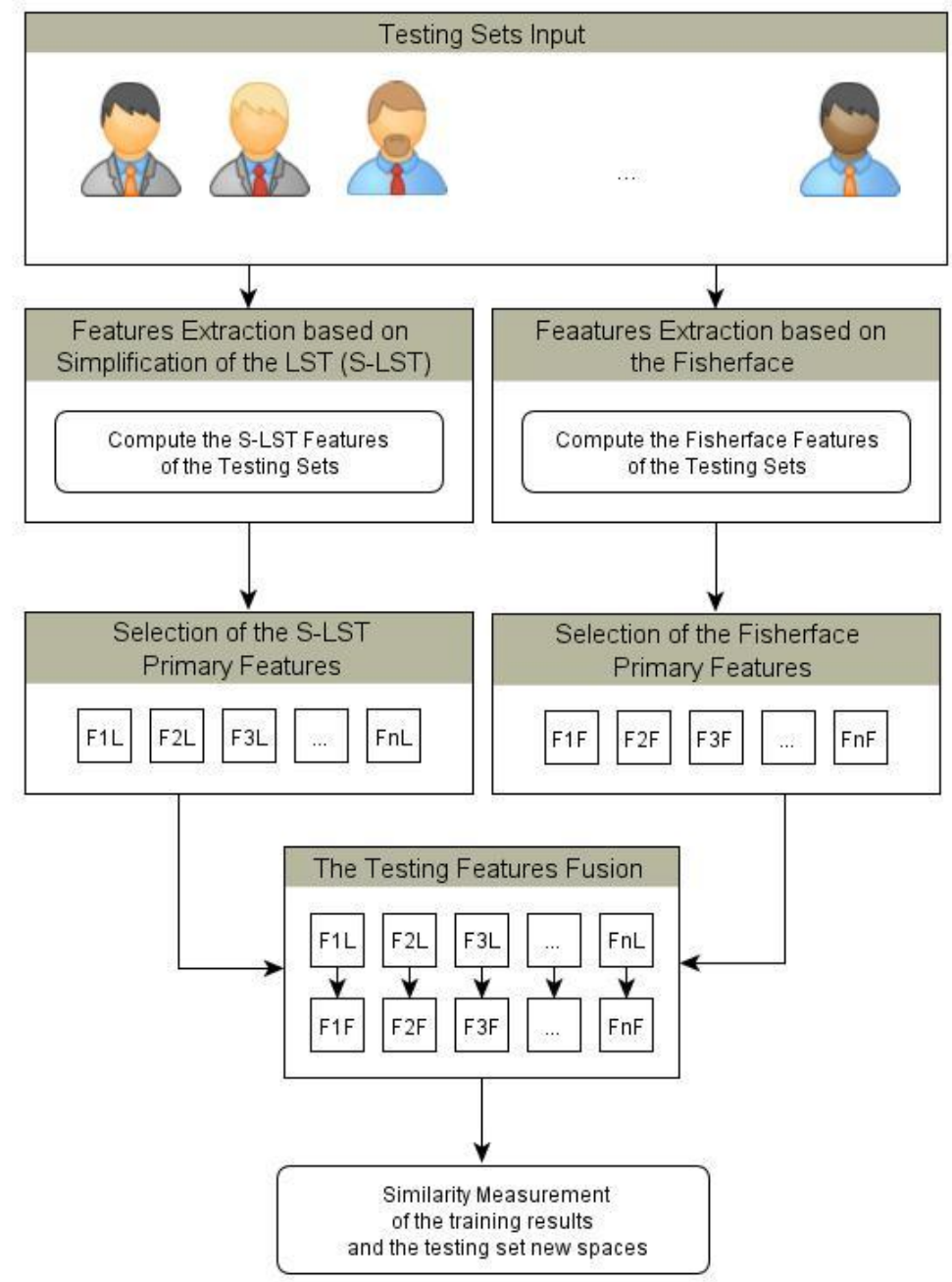

Figure 2. Testing Process of the Proposed Model

The optimum value of the LST $\left(L_{2}\right)$ can be computed by summation of the $L_{1}$ matrix as follows

$$
L_{2}(1, \mathrm{col})=\sum_{\text {row }=1}^{n} L_{1}(\text { row }, \text { col })
$$

The subtraction result of the $L_{2}$ and $L_{l}$ is the optimum values of the LST as written in Pseudocode 4 . The matrix sizes produced by Equation (1), (2), (3), and 4 are huge sizes. However, these are computation time problem. Therefore, the matrix must be changed in the sparse matrix form to reduce computation time. The matrix element just saves the " 1 " values based on the location. 


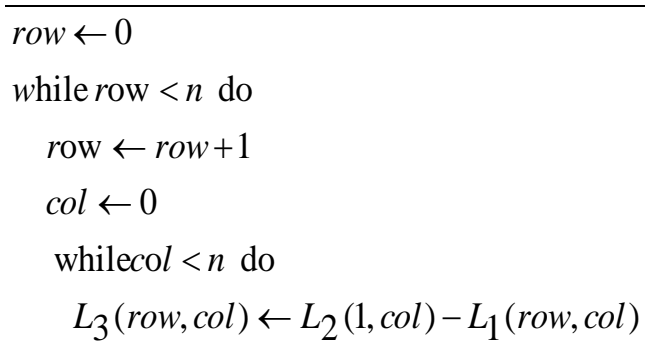

End while of $\mathrm{col}$

End while of row

Pseudo-code 4. It is used to create $L_{3}$

C. Simplification of the Laplacian Smoothing Transform (S-LST)

The S-LST involved the Neuman Discrete and Kronecker Matrix to appear the features. It can be calculated by using the summation of the Neuman Discrete Matrix and the maximum image feature values as follows

$$
\begin{aligned}
& N_{\text {height }, \text { width }}=\Delta_{\text {height }, \text { width }}+L_{4} \\
& L_{4}(\text { row }, \text { row })=\left\{\begin{array}{lc}
-1 & \text { if row }=1 \text { or } \text { row }=\frac{n}{2} \\
-2 & \text { otherwise }
\end{array}\right.
\end{aligned}
$$

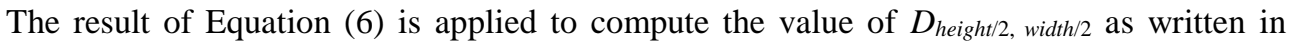
Equation (7).

$$
\frac{D_{\text {height }}}{2}, \frac{\text { width }}{2}=\frac{1}{2 \times n}\left(L_{4}(\text { row }, \text { row })\right)
$$

Furthermore, the value of $\Delta_{h, w}$ can be calculated by using the Kronecker matrix operation $(\otimes)$ as expressed in Equation (8).

$$
\Delta_{\text {height }, \text { width }}=D_{\frac{\text { height }}{2}, \frac{\text { width }}{2}} \otimes \tilde{I}+\tilde{I} \otimes D_{\frac{h e i g h t}{2}}, \frac{\text { width }}{2}
$$

In this case, $\tilde{I}$ represents the matrix with two rows and two columns, where all of the elements are 1. The results of the Equation (8) and its transpose are selected the maximum values as shown in Equation (9)

$$
\Delta_{\text {height }, \text { width }}=\max \left(\Delta_{\text {height }, \text { width }},\left(\Delta_{\text {height }, \text { width }}\right)^{T}\right)
$$

\section{Compute the new spaces of the $S$-LST}

The calculation results of the S-LST as written in the Equation (5) are applied to obtain the characteristics of the object. It can be achieved by the Eigenvalue $(\lambda)$ and Eigenvector $(\Lambda)$ computation. The characteristic equation can be written as follows 


$$
\begin{aligned}
& \hat{\lambda} \times A=N_{\text {height }}, \text { width } \\
& \times A \\
& N_{\text {height }, \text { width }} \times A-\hat{\lambda} \times A=0 \\
& \left(N_{\text {height }, \text { width }}-\hat{\lambda}\right) \times A=0 \\
& \left(N_{\text {height }, \text { width }}-\hat{\lambda} \times I\right) \times A=0 \\
& \left|N_{\text {height }, \text { width }}-\hat{\lambda} \times I\right|=0
\end{aligned}
$$

The results of Equation (10) delivered the Eigenvalue and the Eigenvector, where the Eigenvalues are decreasingly ordered as mentioned in Equation (11) and followed the Eigenvectors shifting based on the corresponding column position to obtain the primary features as written in the Equation (12)

$$
\begin{aligned}
& \hat{\lambda}_{1} \geq \hat{\lambda}_{2} \geq \hat{\lambda}_{3} \geq \hat{\lambda}_{4} \geq \cdots \hat{\lambda}_{m-1} \geq \hat{\lambda}_{m} \\
& \hat{\Lambda}_{n, 1}, \hat{\Lambda}_{n, 2}, \hat{\Lambda}_{n .3}, \hat{\Lambda}_{n, 4}, \cdots \hat{\Lambda}_{n, m-1}, \hat{\Lambda}_{n, m} .
\end{aligned}
$$

The result of equation (12) must be multiplied by the training set or the testing sets to acquire the new spaces of the S-LST. The new spaces produced are the characteristics of the object. They are applied to measure the similarity among objects as follows

$$
F_{T r_{-} L S T}=T r_{1, n} \times \hat{\Lambda}_{n, N o F}
$$

\section{E. Selection of the S-LST Primary Features}

For each image produced the new spaces on the row vector form, however, the problem is high dimensionality of the $F_{T r}$ as shown in Equation (14). The more top dimensionality of the features produced, the longer time spent to measure the similarity. To overcome this problem, the proposed model only takes the primary features for measurement. In this case, this size is $c$ 1 , where $c$ represents classes. As known, the image dimensionality is high, which is $n$. The proposed model removed some features, which is $b=n-(c+1)$ features, in this case, $n>>c$. The selection of the primary features can be described in Figure 3.

$$
F_{T r_{-} L S T}=\left[\begin{array}{llll}
F L(1) & F L(2) & \cdots & F L(n)
\end{array}\right]
$$

The Equation (14) produces the features, but they have high dimensionality. Figure 3 showed that the proposed model selected principal features and removed unnecessary features. The main features are taken until $c-1$. The selection of the main features reduced a lot of computation times when the similarity measurements should be completed.

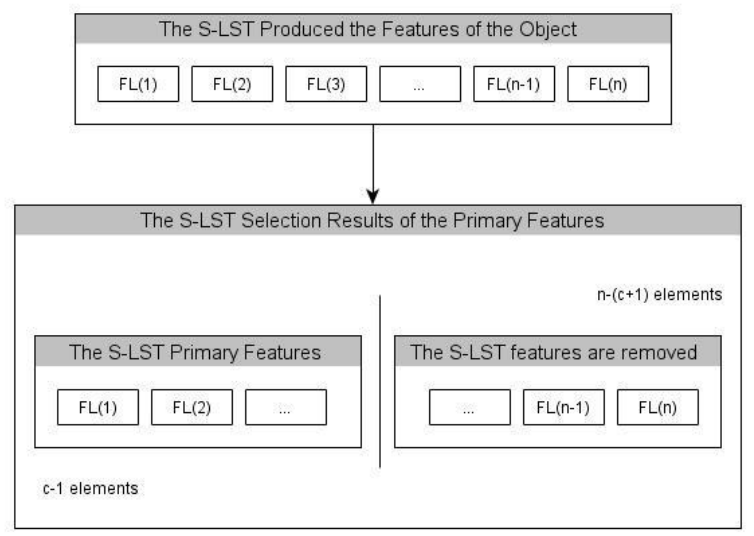

Figure 3. Selection of the S-LST Primary Features 


\section{F. Features Extraction based on the Fisherface}

The proposed model also employed feature extraction based on the derivative of the LDA (Fisherface), the feature extraction results are utilized to augment the object characteristics so that the object is more accessible to be classified. In this case, the training sets features are reduced until $c-1$ the features. Furthermore, the features extraction results of the Fisherface and the S-LST are united to compose the new spaces. The Fisherface can maximize the different classes mean distance so that the object of the various classes can be readily distinguished. The Fisherface can also select basis vectors based on how to choose the two categories or more can be well separated. It is different with Eigenface. The Eigenface can only maximize its variation and determine the first basis vector. Principally, the Fisherface performed to maximize the criterion function as follows

$$
J(W)=\frac{\sum_{\text {class }=1}^{c}\left|\hat{\mu}_{\text {class }}-\hat{\mu}\right|^{2}}{\sum_{\text {class }=1}^{c} \hat{S}_{\text {class }}^{2}}
$$

Where

$$
\begin{aligned}
& \hat{\mu}=w^{T} \times \mu \\
& \hat{\mu}_{\text {class }}=w^{T} \times \mu_{\text {class }}
\end{aligned}
$$

\section{G. Create Data Input of the Fisherface}

The Fisherface process can be commenced by calculating of the Principal Component Analysis (PCA). It produces the weight and projection matrix. The input of the Fisherface $(\hat{I})$ can be calculated by multiplication of the zero mean $(Z)$ and the PCA projection matrix $(P)$ as follows

$$
\hat{I}=Z \times P
$$

The Fisherface can find the informative projection using label information. It can be used to maximize the component axes for class separation. The Fisherface can linearly separate the data, and minimize the projection results as much as $s=n-(c+1)$. It means that the Fisherface transform the data input onto $c-1$ dimensions, where $m$ represents classes.

\section{H. Compute Between and Within Class Scatter}

The value of Between Class Scatter $\left(S_{B}\right)$ and Within Class Scatter $\left(S_{W}\right)$ play an essential role in features extraction. The bigger the value of $S_{B}$, the easier class separated. Principally, the Fisherface maximizes the value of $S_{B}$ and minimizes the value of $S_{W}$ as the following equation:

$$
\begin{aligned}
\frac{d}{d w} J(W) & =0 \\
0 & =\frac{d}{d w}\left(\frac{w^{T} \times S_{B} \times w}{w^{T} \times S_{w} \times w}\right) \\
0 & =S_{B} \times w-J(w) \times S_{W} \times w
\end{aligned}
$$




$$
\begin{aligned}
w^{*} & =\operatorname{Arg} \max J(w) \\
& =\left(\frac{w^{T} \times S_{B} \times w}{w^{T} \times S_{W} \times w}\right)
\end{aligned}
$$

Calculating of $S_{B}$ involved mean of the class and all data sets, whereas the $S_{W}$ uses mean of the class only as follows

$$
\begin{aligned}
& S_{B}=\sum_{\text {class }=1}^{c} N_{\text {class }} \times\left(\mu_{\text {class }}-\mu\right) \times\left(\mu_{\text {class }}-\mu\right)^{T} \\
& S_{w}=\sum_{\text {class }=1}^{c} \frac{1}{\left(N_{\text {class }}-1\right)} \times \sum_{x \in \omega_{\text {class }}}\left(x-\mu_{c}\right) \times\left(x-\mu_{c}\right)^{T}
\end{aligned}
$$

\section{Compute the Eigenvector and the new spaces of the Fisherface}

The key features of the objects can be found by calculating the Eigenvector and Eigenvalue. They can be computed from Argmax $J(w)$ as mentioned in Equation (18), (19), (20), and (21). The results of $S_{B} / S_{W}$ are an orthogonal matrix, where it has the inverse result is equal to its transpose. Furthermore, the primary characteristics of $J(w)$ can be found as follows

$$
\begin{aligned}
& \lambda \times A=\left(\frac{S_{B}}{S_{w}}\right) \times A \\
& \left(\frac{S_{B}}{S_{w}}\right) \times A-\lambda \times A=0 \\
& \left(\frac{S_{B}}{S_{w}}-\lambda\right) \times A=0 \\
& \left(\frac{S_{B}}{S_{w}}-\lambda \times I\right) \times A=0 \\
& \left|\frac{S_{B}}{S_{w}}-\lambda \times I\right|=0
\end{aligned}
$$

In this case, the value of $I$ represents identity matrix, where the dimensionality of identity matrix has the same size as the $S_{B}$. The results of Equation (22) are the Eigenvalues and Eigenvectors. Hereafter, the Eigenvalues must be decreasingly ordered and followed by the Eigenvector columns. The results of the sorting are shown as follows

$$
\begin{aligned}
& \tilde{\lambda}_{1} \geq \tilde{\lambda}_{2} \geq \tilde{\lambda}_{3} \geq \tilde{\lambda}_{4} \geq \tilde{\lambda}_{5} \geq \cdots \tilde{\lambda}_{m-1} \geq \tilde{\lambda}_{m} \\
& \tilde{\Lambda}_{n, 1}, \tilde{\Lambda}_{n, 2}, \tilde{\Lambda}_{n, 3}, \tilde{\Lambda}_{n, 4}, \cdots \tilde{\Lambda}_{n, m-1}, \tilde{\Lambda}_{n, m} .
\end{aligned}
$$

The principal features of the Fisherface are acquired by multiplication of the training sets and the Eigenvectors as mentioned in Equation (24). The results of the primary features produce the new spaces as follows

$$
F_{T r_{-} F h}=T r_{1, n} \times \tilde{\Lambda}_{n, N o F}
$$




\section{J. Selection of the Fisherface Primary Features}

The operation results as mentioned in Equation (25) produce features with the higher dimensionality. It can give the impact on the computation time. The more top dimensionality provided, the more extended computation time spent. Therefore, the results must be taken the essential features. They are applied to capture the object characteristics which is $c-1$ features as displayed in Figure 4. It showed that there were a lot of the worthless features. They must be removed to reduce the computation time and increase the recognition results.

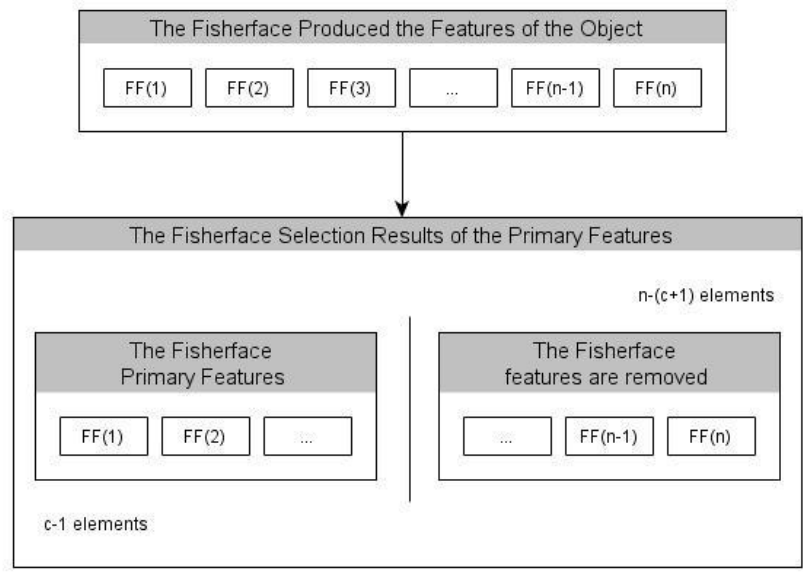

Figure 4. Selection of the Fisherface Primary Features

\section{K. The Training Features Fusion}

Features fusion processes are to join the S-LST and Fisherface principal features, where the join processes are conducted to vertical unity the essential features produced by the S-LST and Fisherface. The main features can distinguish the objects, where the unique features will influence the results of recognition. The S-LST can preserve the particular global information and the local feature areas on the LST of the rows and columns so that they can represent primary characteristics of the object. The Fisherface is the derivative of the LDA $(J(w))$, where the value of the $S_{B}$ can be maximized the results so that the information produced is more reliable and stable. The fusion of features models is vertically composed of the corresponding feature elements between the S-LST and Fisherface as seen in Figure 5. The Features fusion can strengthen the object characteristics to be classified.

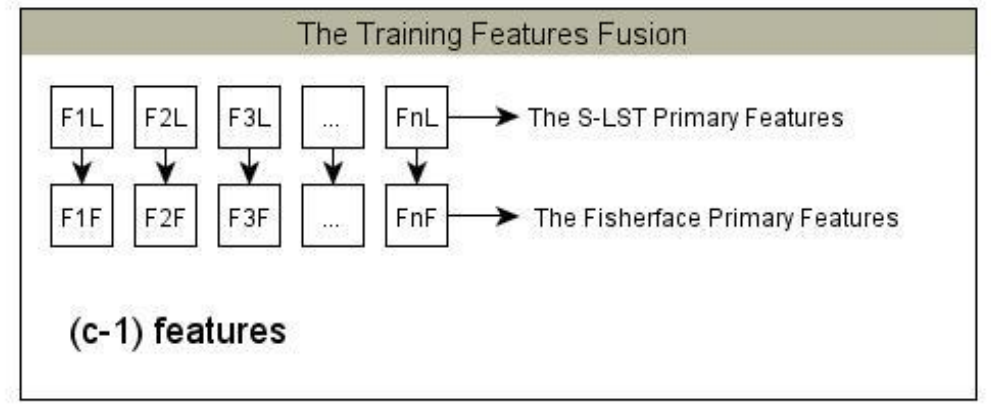

Figure 5. The Training Features Fusion (FF) of the S-LST and Fisherface

\section{Face Image Similarity Measurement}

Figure 2 displayed the features extraction on the testing process. However, it is different to the features extraction on the training sets. The testing process used the results of the training process to obtain the primary features before the measurement of the training and testing features is performed. In this process, the S-LST features have been achieved by the multiplication of the 
testing sets and the new projection of the S-LST, whereas the Fisherface features were computed through multiplication of the testing sets and the new projection of the Fisherface. Furthermore, the primary features were selected based on the most significant Eigenvalues and corresponding to the eigenvector, and followed by the main features fusion of them which are $c$ - 1 features, where $c$ represents classes of the training sets. The features extraction results of the training and the testing sets have measured the similarity to classify the testing image. In this case, the similarity measurement used Manhattan method as follows

$$
d=\sum_{f e a=1}^{c-1} \sum_{m e t=1}^{2} \mid F F_{f e a, m e t}-F T_{f e a}, \text { met } \mid
$$

\section{Databases and Scenarios}

In this research, three different databases were selected to evaluate the robustness of the proposed model, i.e., the ORL, University of Bern, and YALE databases as displayed in Figure 6. It presented three databases with four different poses, which is the ORL on the first row, the University of Bern on the second row, and the YALE on the last row.

The ORL database was captured from April 1992 until April 1994 by AT\&T Cambridge University Computer Laboratory. Forty persons were taken with different poses, expressions, and accessories. Ten different poses, expressions and accessories were used as a reference to capture for each person, so four hundred images were available on the ORL database [24].

The last database is the YALE database. It has one hundred and sixty-five samples. They were taken from fifteen persons with eleven different poses, expressions, an angle of view, and lightings. The images were also cropped from the original images onto 136x104 pixels [25].

The University of Bern database is well known as "UoB database." It is taken from thirty persons with ten different poses, but the same expressions, thus the total number are three hundred images. The images used as experimental are the cropping results 140x120 pixels of the original image 512x342 pixels [26].

Several scenarios have applied the evaluation of the proposed model. The proposed model evaluations are randomly conducted with 10 -folds cross-validation for all databases. The different primary features were sequentially applied in similarity measurements so that the proposed model can be measured the reliability of the testing sets used as depicted in Table 1. The remaining of images was utilized as the testing sets. In Table 1, the symbol of $G X$ represents the number of image gallery as the training sets ( $X$ is the positive number), for example, $G 3$ means the training sets utilized three images. $P Y$ represents number of the testing sets $(Y$ is the positive number), such as $P 7$ means the testing sets applied seven images.

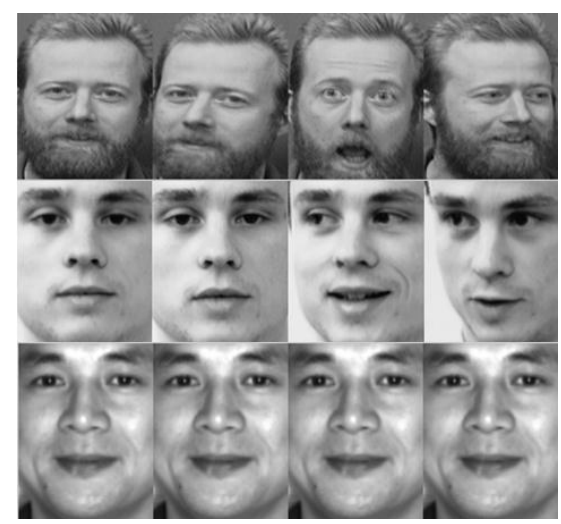

Figure 6. The Image Samples of the ORL [24], YALE [25], and UoB [26] Database 
Table 1. Experimental Scenario

\begin{tabular}{cccc}
\hline \multirow{2}{*}{ Scenario } & \multicolumn{3}{c}{ Face Image Database } \\
\cline { 2 - 4 } & ORL & UoB & YALE \\
\hline $1^{\text {st }}$ & $G 5 / P 5$ & $G 5 / P 5$ & $G 5 / P 6$ \\
$2^{\text {nd }}$ & $G 4 / P 6$ & $G 4 / P 6$ & $G 4 / P 7$ \\
$3^{\text {rd }}$ & $G 3 / P 7$ & $G 3 / P 7$ & $G 3 / P 8$ \\
$4^{\text {th }}$ & $G 2 / P 8$ & $G 2 / P 8$ & $G 2 / P 9$ \\
\hline
\end{tabular}

For each database employs 20 until 39 features, 10 until 29 features, and 10 until 14 features for ORL, UoB, and YALE

\section{Experimental Results And Discussions}

Three databases as mentioned in Table 1 were applied to perform the training and testing processes. The proposed model completed the training process using two to five images for the first until the fourth scenario. The proposed model was also assessed by using the different features based on the cross-validation. If the proposed model used the ORL, then features applied are greater than nineteen and less than forty. If the proposed model utilized is the UoB, then ten until twenty-nine features are employed for the measurements. If the database used is the YALE, then features used are greater than or equal to ten and less than or equal to fourteen.

\section{A. Experimental Results on the ORL Database}

In this research, twenty times approaches were conducted, which are using twenty to thirtynine features. For each experiment was randomly selected the training sets, it is performed ten times (10-folds cross-validation). The experimental results exhibited that the proposed model produced the highest accuracy when five training sets are applied to extract the primary features, which is $98.7 \%$ recognition rate, even the proposed model also provided the smallest standard deviation for the first scenario. The results showed definitely that the proposed model could recognize the testing samples with the minor errors. On the second scenario, the proposed model produced $95.5 \%$ recognition rate and 0.8 standard deviations. It demonstrated that the S-MFA, S-LPP, and S-LDA surpassed the proposed model, which is 0.3 . However, the proposed model also proved that standard deviation delivered is smaller than the others, including the S-MFA, SLPP, and S-LDA (see Table 2).

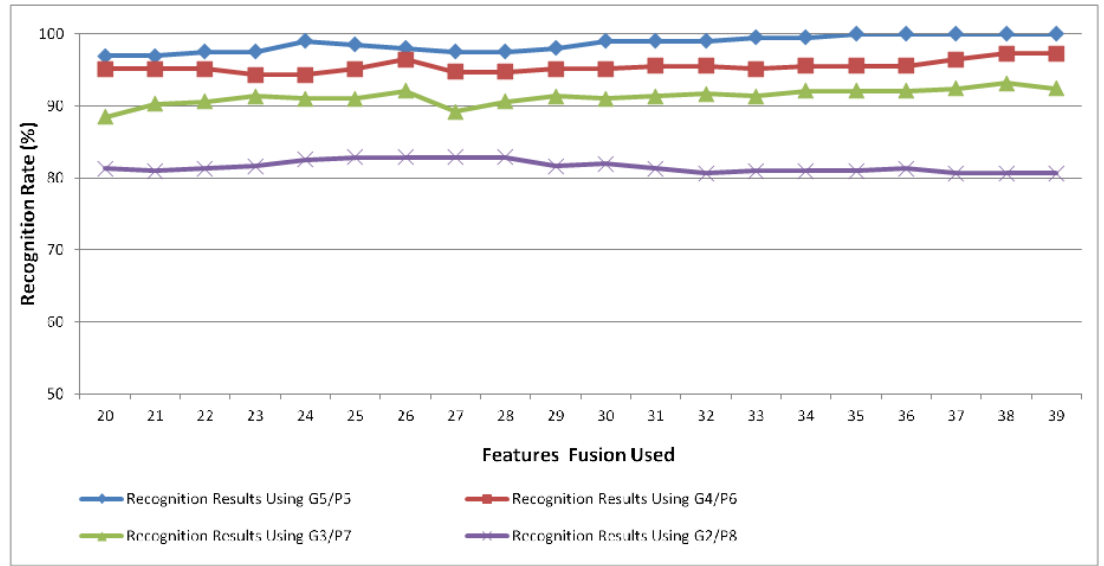

Figure 7. Recognition Results Based on the Feature Fusion of the S-LST and Fisherface on the ORL Database

Table 2 indicated that the proposed model is better than the other methods. The similar condition also occurred in the third and the fourth scenarios. The average of overall experimental results as depicted in Figure 7. It demonstrated the highest accuracy happened when five training sets are applied. The lowest recognition rate appeared when the least training sets are used. In 
detail, Figure 7 demonstrated the average of the recognition rate. The features fusion was shown clearly, the proposed model preserve the information of the rows and columns can increase and produce the higher recognition rate than LDA and LST+LDA.

Table 2. Comparison of the Different Methods on the ORL Database

\begin{tabular}{ccccc}
\hline Methods & \multicolumn{4}{c}{ Scenarios } \\
& G5/P5 & G4/P6 & G3/P7 & G2/P8 \\
\cline { 2 - 5 } & $98.7 \pm 1.1$ & $95.5 \pm 0.8$ & $91.3 \pm 1.1$ & $81.6 \pm 0.8$ \\
\hline Proposed Model & $97.2 \pm 1.3$ & $95.8 \pm 1.3$ & $92.3 \pm 1.7$ & $85.2 \pm 2.2$ \\
S-LPP [27] & $96.9 \pm 1.2$ & $95.0 \pm 1.6$ & $92.1 \pm 1.8$ & $84.5 \pm 2.9$ \\
LST+LDA [13] & $97.4 \pm 1.2$ & $95.8 \pm 1.5$ & $92.4 \pm 1.3$ & $84.9 \pm 2.3$ \\
S-MFA & $96.9 \pm 0.9$ & $95.4 \pm 1.2$ & $92.3 \pm 1.7$ & $84.8 \pm 2.3$ \\
S-NPE & $97.2 \pm 1.3$ & $95.8 \pm 1.3$ & $92.3 \pm 1.7$ & $85.2 \pm 2.2$ \\
S-LDA & $93.0 \pm 1.9$ & $90.3 \pm 1.7$ & $86.0 \pm 2.0$ & $77.6 \pm 2.5$ \\
LPP & $94.3 \pm 1.4$ & $91.6 \pm 1.9$ & $86.1 \pm 1.9$ & $75.5 \pm 3.3$ \\
LDA & $87.9 \pm 2.5$ & $84.2 \pm 2.1$ & $78.9 \pm 2.3$ & $70.7 \pm 2.7$ \\
PCA & & &
\end{tabular}

B. Experimental Results on the YALE Database

In this experiment, the similarity measurements only used ten to fourteen features, because the YALE database has fifteen classes, so maximum features used is fourteen. The proposed model showed the best performance on the YALE database. In detail, Figure 8 demonstrated the average of recognition rate results of the 10 -folds cross-validation.

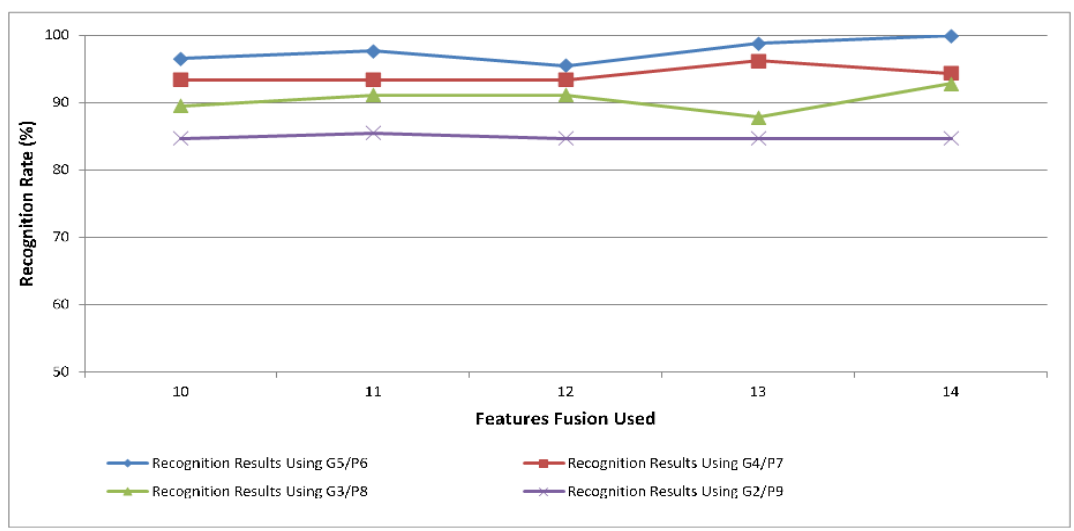

Figure 8. Recognition Results Based on the Feature Fusion of the S-LST and Fisherface on the YALE Database

Table 3. Comparison of the Different Methods on the YALE Database

\begin{tabular}{ccccc}
\hline \multirow{2}{*}{ Methods } & \multicolumn{4}{c}{$\begin{array}{c}\text { Scenarios } \\
\text { Gecognition Rate } \pm \text { Standard Deviation) }\end{array}$} \\
\cline { 2 - 5 } & G5/P6 & G4/P7 & G3/P8 & G2/P9 \\
\hline Proposed Model & $97.7 \pm 1.7$ & $94.1 \pm 1.2$ & $90.5 \pm 1.9$ & $84.9 \pm 0.3$ \\
S-LPP [27] & $81.4 \pm 2.9$ & $76.0 \pm 3.4$ & $72.0 \pm 4.0$ & $57.9 \pm 4.5$ \\
LST+LDA [13] & $96.1 \pm 1.1$ & $93.9 \pm 2.3$ & $90.2 \pm 2.8$ & $84.0 \pm 3.6$ \\
S-MFA & $81.1 \pm 3.1$ & $76.9 \pm 3.1$ & $71.2 \pm 4.0$ & $57.2 \pm 4.3$ \\
S-NPE & $80.9 \pm 3.5$ & $77.0 \pm 3.4$ & $71.9 \pm 3.9$ & $57.5 \pm 4.7$ \\
LPP & $75.8 \pm 4.6$ & $72.7 \pm 4.2$ & $67.2 \pm 4.1$ & $54.5 \pm 5.2$ \\
S-LDA & $81.7 \pm 3.2$ & $77.8 \pm 3.0$ & $72.3 \pm 4.4$ & $57.6 \pm 4.1$ \\
LDA & $76.9 \pm 3.2$ & $73.0 \pm 5.4$ & $62.3 \pm 4.5$ & $45.7 \pm 4.2$ \\
PCA & $57.7 \pm 3.8$ & $55.7 \pm 3.5$ & $50.0 \pm 3.5$ & $46.0 \pm 3.4$ \\
\hline
\end{tabular}


Figure 8 also displayed the relation of the features fusion used, recognition rate, and several training sets applied. However, the more usage of the training sets are produced, the higher acceptance rate is delivered. In this case, the proposed model is superior to the other methods, i.e., LST-LDA, S-MFA, S-NPE, S-LPP, LPP, S-LDA, LDA, and PCA for all scenarios as displayed (see Table 3). On the first scenario, the difference between the proposed model and LST+LDA in recognition is $1.6(97.7-96.1=1.6)$, whereas in standard deviation is $0.6(1.7-1.1=$ $0.6)$. It indicated that the proposed model is superior to LST+LDA as much as $1(1.6-0.6=1)$. The second, third and last scenarios are also better than the others. The best recognition rate obtained is $97.7 \%$, where five poses are applied as the training sets.

\section{Experimental Results on the UoB Database}

The last scenario, twenty experiments were also conducted in this database, but it is different with the first scenario. In this case, the similarity measurements utilized ten to twenty-nine features, because the UoB database has thirty classes, whereas the ORL database used twenty to thirty-nine features for similarity measurements. The results of experiments were demonstrated in Figure 9. The results displayed four scenarios, where the first scenario outperformed to the second, third, and the last scenario. The best and average results occurred on the first scenario, which is $95.33 \%$ and $94.53 \%$. This result is equal to SDM-2DLDA method. However, the proposed model has performed better than the others, i.e., PCA, LDA, LPP, and O-LPP (see Table 4). The similarity of the testing samples on the different class caused misclassification.

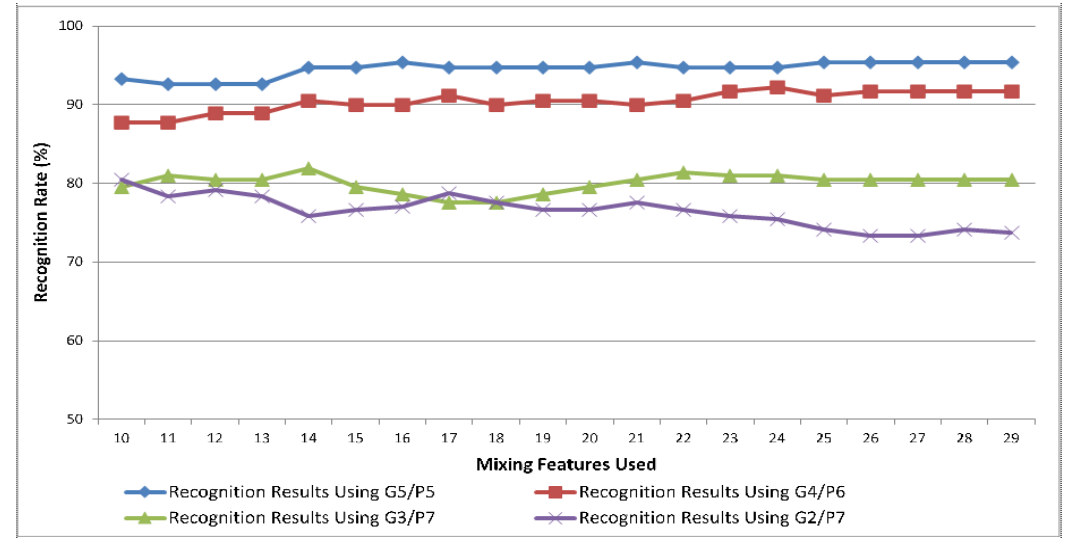

Figure 9. Recognition Results Based On the Feature Fusion of the S-LST and Fisherface on the UoB Database

Table 4. Comparison of the different methods on the UoB database

\begin{tabular}{ccccc}
\hline \multirow{2}{*}{ Methods } & \multicolumn{4}{c}{ Scenarios } \\
& G5/P5 & G4/P6 & G3/P7 & G2/P8 \\
\cline { 2 - 5 } & 94.53 & 90.56 & 80.04 & 76.47 \\
Average of the Proposed Method & 95.33 & 92.2 & 81.9 & 80.4 \\
Maximum of the Proposed Method & 92 & 83.33 & 71.43 & 66.25 \\
PCA & 91.33 & 81.67 & 71.43 & 66.67 \\
LDA & 95.33 & 85 & 74.76 & 67.92 \\
SDM-2DLDA & 90 & 86.67 & 71.9 & 67.5 \\
LPP & 89.33 & 77.22 & 66.19 & 63.33 \\
O-LPP & & & & \\
\hline
\end{tabular}

However, the proposed model also has weakness in recognition, because of an appearancebased features extraction. If the testing images have a similar appearance to the other class of the training sets, then the recognition results have tended misclassification. 


\section{Conclusions}

Overall, the proposed model performed the feature fusion results to recognize the testing image. It is also evaluated by using the ORL, YALE and UoB database. The results reveal that the proposed model obtained higher performance than the others, i.e., PCA, LDA, S-LDA, SMFA, S-NPE, LPP, O-LPP, and even LST-LDA. It also showed the best performance when the proposed model is employed to the ORL database, which is $100 \%$ recognition rate in several time experiments. The similar condition also appeared on the YALE database. The proposed model delivered an excellent performance when five images are employed to the training sets. It means that the fusion of the S-LST and Fisherface can be well implemented on the face recognition.

\section{References}

[1]. Kim, C. Choi, Image covariance-based subspace method for face recognition, Pattern Recognition 40(5), pp. 1592-1604, (2007)

[2]. S.M. Huang, J.F. Yang, Improved principal component regression for face recognition under illumination variations, IEEE Trans. Signal Process. Lett., 19(4), pp.179-182, (2012)

[3]. H. Lu, K.N. Plataniotis, A.N. Venetsanopoulos, MPCA: multilinear principal component analysis of tensor objects, IEEE Transactions on Neural Networks, 19(1), (2008).

[4]. Gumus, N. Kilic, A. Sertbas, Evaluation of face recognition techniques using PCA, wavelets and SVM, Expert Syst. Appl. 37(9), pp. 6404-6408, (2010).

[5]. G.F. Lu, J. Zou, Y. Wang, Incremental complete LDA for face recognition, Pattern Recognit. 45(7) pp. 2510-2521, (2012)

[6]. X. Shu, Y. Gao, H. Lu, Efficient linear discriminant analysis with locality preserving for face recognition, Pattern Recognit., 45(5), 1892-1898, (2012)

[7]. A.M. Martínez, A.C. Kak, PCA versus LDA, IEEE Trans. Pattern Anal. Mach. Intell., 23(2), pp. 228-233, (2001)

[8]. Muntasa, A., Facial recognition using square diagonal matrix based on two-dimensional linear discriminant analysis. Int. Rev. Comput. Software (I.RE.CO.S.), 10(7), pp. 718-725, (2015a)

[9]. Muntasa, A., "New modelling of modified two dimensional Fisherface based feature extraction", Telkomnika 12(1), pp. 115-122, (2014)

[10]. O. Deniz, M. Castrillon, M. Hernandez, Face recognition using independent component analysis and support vector machines, Pattern Recognit. Lett. 24, pp. 21532157, (2003)

[11]. X. He, S. Yan, Y. Hu, H. Zhang, Learning a locality preserving subspace for visual recognition, in: Proceedings of Ninth International Conference on Computer Vision, France, pp. 385-392, (October 2003)

[12]. Muntasa, A., A new approach: The local feature extraction based on the new regulation of the locally preserving projection, Applied Math. Sci., 9 (102), pp. 5065-5078, (2015b)

[13]. GU Sui Cheng, TAN Ying, and HE Xin Gui, "Laplacian Smoothing Transform for face recognition”, Science China Information Sciences, 53(12), pp. 2415-2428, (2010)

[14]. J.-H. Park, Z. Zhang, H. Zha, and R. Kasturi, "Local smoothing for manifold learning," In IEEE Computer Society Conference on Computer Vision and Pattern Recognition, Vol. 2, pp. 452-459, (2004)

[15]. Muntasa, A., The Human Facial Expression Classification Using the Center Kernel Subspace based the Ridge Regression, Journal of Computer Science, 11(11), pp. 1054$1059,(2015 \mathrm{c})$

[16]. Z. Zhang, H. Zha, Principal manifolds and nonlinear dimensionality reduction via tangent space alignment, SIAM J. Sci. Comput., 26(1), pp. 313-338, (2004)

[17]. J. Gui, Z. Sun, W. Jia, Discriminant sparse neighborhood preserving embedding for face recognition, Pattern Recognit. 45(8), pp. 2884-2893, (2012) 
[18]. Y.Z. Goh, A.B.J. Teoh, K.O.M. Goh, Wavelet-based illumination invariant pre-processing in face recognition, Journal of Electronic Imaging 18(2), pp. 1-12, (2009)

[19]. S. Yan, D. Xu, B. Zhang, H.-J. Zhang, Q. Yang, S. Lin, Graph embedding and extension: a general framework for dimensionality reduction, IEEE Transactions on Pattern Analysis and Machine Intelligence 29(1), pp. 40-51, (2007)

[20]. Y. Xu, D. Zhang, J. Yang, J. Yang, An approach for directly extracting features from matrix data and its application in face recognition, Neurocomputing, 71 (10-12), pp. 1857-1865, (2008)

[21]. W. Yu, Z. Wang, W. Chen, A new framework to combine vertical and horizontal information for face recognition, Neurocomputing 72 (4-6), pp. 1084-1091, (2009)

[22]. X. Chai, S. Shan, X. Chen, W. Gao, Locally linear regression for pose-invariant face recognition, IEEE Transactions on Image Processing 16(7), pp. 1716-1725, (2007)

[23]. Muntasa, A., Homogeneous and Non-homogeneous Polynomial Based Eigenspaces to Extract the Features on Facial Images, Journal of Information Processing System 12(4), pp. 591-611, (2016)

[24]. The Database of Faces (formerly 'The ORL Database of Faces') [Online]. Available: http://www.cl.cam.ac.uk/research/dtg/attarchive/facedatabase.html.

[25]. The Yale Face [Online]. Aatabase Available: http://cvc.cs.yale.edu/cvc/projects/yalefaces/yalefaces.html.

[26]. IAM Faces Database [Online]. Available: http://www.fki.inf.unibe.ch/databases/iamfaces-database

[27]. Cai D, He X, Hu Y, et al, "Learning a spatially smooth subspace for face recogni-tion," In: IEEE Conf. Computer Vision and Pattern Recognition (CVPR07), Min-neapolis, MN, USA, pp. 1-7, (2007)

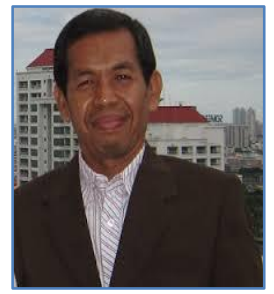

Arif Muntasa received the bachelor degree in Mathematics, ITS, Surabaya, Indonesia, 1993. He received both Master of Engineering and Doctor of Philosophy degrees in Electrical Engineering Department, ITS, Surabaya, Indonesia, 1998 and 2010 respectively. Currently, he is lecturer of Informatics Engineering Department, University of Trunojoyo Madura, Bangkalan, Indonesia. His research interest is computer vision, pattern recognition, and computational intelligence. 